Figure 1. Graphic 1. This graphic shows the frequence in percentage \% of contraceptive methods used by Rheumatoid Arthritis (RA) $n=56$ and Systemic Lupus Erythematosus (SLE) $n=35$ patients, which are categorized by the grade of effectiveness. Only were included patients that had started sexual activity.
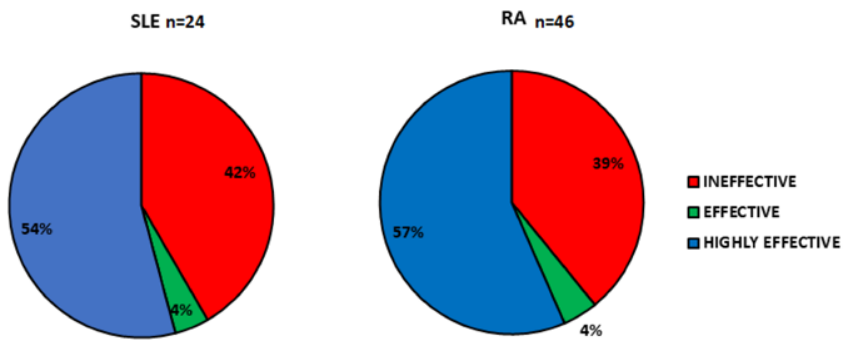

Figure 2. Graphic 2. This graphic shows the percentage of use according to the effectiveness of contracetive methods. RA, Rheumatoid Arthritis; SLE, Systemic Lupus Erythematosus. Only were included patients that had started sexual activity.

Disclosure of Interests: None declared

DOI: 10.1136/annrheumdis-2020-eular.6324

\section{SAT0578 $\quad$ RHEUMAL REGISTRY PROVIDES FIRST INSIGHTS INTO MUTUAL INTERDEPENDENCIES BETWEEN RHEUMATIC AND MUSCULOSKELETAL DISEASES AND MALIGNANCIES}

K. Benesova ${ }^{1}$, L. Diekmann ${ }^{1}$, M. Czaja ${ }^{1}$, K. Jordan ${ }^{1}$, J. Leipe ${ }^{2}$, H. M. Lorenz ${ }^{1}$. ${ }^{1}$ University Hospital Heidelberg, Department of Medicine V, Hematology, Oncology and Rheumatology, Heidelberg, Germany; ${ }^{2}$ University Hospital Mannheim, Division of Rheumatology, Department of Medicine V, Mannheim, Germany

Background: Knowledge about interdependencies between rheumatic and musculoskeletal diseases (RMDs) and malignancies is limited on the clinical and molecular level. Particularly, valid prospective data on the timely association of malignancies in patients with RMDs and treatment of the latter are sparse.

Objectives: Due to the heterogeneous patient population, a registry-based study has been conducted in order to provide insights into mutual interdependencies and novel evidence for suitable clinical management of patients with concomitant RMD and malignancies.

Methods: The RheuMal registry is a long-term, open-end observational study designed to address the specific situation of patients suffering from concomitant RMD and concomitant malignancy and/or premalignant conditions. The RheuMal registry is one of the three subregistries of the MalheuR project, a registry-based study initiated in July 2018 at the at the university hospital Heidelberg, Germany. Results: Data from the RheuMal registry $(n=404)$ show an earlier onset of gender-specific cancers and malignant melanoma in RMD patients compared to data from the German Cancer Registry Data of the Robert-Koch-Institute: compared to the reference population, in RMD patients breast cancer $(n=32)$ occurred 5.3 years and prostate cancer $(n=16) 3.3$ years earlier. Onset of malignant melanoma was 2.4 years earlier in females $(n=9)$ and 1.1 years in males $(n=7)$ with concomitant RMD. The mean latency between the initial diagnosis of the RMD and the later occurring malignant condition was 10.2 years. The diagnosis of the malignancy frequently led to a change or interruption of disease-modifying antirheumatic therapy in RMDs.

Conclusion: The RheuMal registry offers first insights into interdependencies between RMDs and malignancies based on demographic data, disease characteristics, clinical management and outcome as well as correlation of specific diagnoses and therapies. The earlier onset of gender-specific cancers and malignant melanoma suggests differences in the epidemiology and course of the malignant disease in RMD patients compared to a healthy reference population, suggesting interdependency between the two disease entities. Future research will focus on further understanding of this interdependency und the underlying molecular mechanisms. Disclosure of Interests: Karolina Benesova Grant/research support from: Study grants for SCREENED study by Abbvie, Novartis and Rheumaliga Baden-Württemberg, Consultant of: One-time participation in Novartis advisory board., Leonore Diekmann: None declared, Maria Czaja: None declared, Karin Jordan Consultant of: Consultancy and/or speaker fees: MSD, Merck, Amgen, Hexal, Riemser, Helsinn, Tesaro, Kreussler, Voluntis, Pfizer, Pomme-med., Jan Leipe Grant/research support from: Consultancy and speaker fees: Abbvie, AstraZeneca, BMS, Celgene, Hospira, Janssen-Cilag, LEO Pharma, Lilly, MSD, Novartis, Pfizer, Roche, Sanofi, UCB. Scientific support: Novartis, Pfizer., Consultant of: Consultancy and speaker fees: Abbvie, AstraZeneca, BMS, Celgene, Hospira, Janssen-Cilag, LEO Pharma, Lilly, MSD, Novartis, Pfizer, Roche, Sanofi, UCB. Scientific support:
Novartis, Pfizer., Speakers bureau: Abbvie, AstraZeneca, BMS, Celgene, Hospira, Janssen-Cilag, LEO Pharma, Lilly, MSD, Novartis, Pfizer, Roche, Sanofi, UCB Hanns-Martin Lorenz Grant/research support from: Consultancy and/or speake fees and/or travel reimbursements: Abbvie, MSD, BMS, Pfizer, Celgene, Medac GSK, Roche, Chugai, Novartis, UCB, Janssen-Cilag, Astra-Zeneca, Lilly. Scientific support and/or educational seminars and/or clinical studies: Abbvie, MSD, BMS Pfizer, Celgene, Medac, GSK, Roche, Chugai, Novartis, UCB, Janssen-Cilag Astra-Zeneca, Lilly, Baxter, SOBI, Biogen, Actelion, Bayer Vital, Shire, Octapharm, Sanofi, Hexal, Mundipharm, Thermo Fisher., Consultant of: see above DOI: 10.1136/annrheumdis-2020-eular.3815

\section{SAT0579 \\ SYSTEMATIC GERIATRIC ASSESSMENT IN OLDER PATIENTS WITH RHEUMATIC DISEASES - THE RHEUMAGIC PILOT STUDY}

A. Berrisch ${ }^{1}$, I. Andreica ${ }^{1}$, S. Tsiami ${ }^{1}$, D. Kiefer ${ }^{1}$, U. Kiltz ${ }^{1}$, X. Baraliakos ${ }^{1}$, J. Braun ${ }^{1}$, B. Buehring ${ }^{1} .{ }^{1}$ Ruhr-University Bochum, Rheumazentrum Ruhrgebiet, Herne, Germany

Background: Current demographic data predict that the number of older adults with rheumatic diseases will considerably increase in the coming years. Geriatric patients differ from younger adults in many ways including their clinical presentation, co-morbidities and response to medication. The management of such patients is often challenging due to the presence of multi-morbidity, polypharmacy and geriatric syndromes (i.e. conditions in which symptoms result from impairments in multiple systems rather than a discrete disease). To systematically assess geriatric patients, specific tools have been developed; however, they are not routinely utilized by rheumatologists. Using these tools could improve patient management and satisfaction in rheumatologic care.

Objectives: To examine the prevalence of 17 common geriatric health problems using validated geriatric assessment tools in older patients with rheumatic and musculoskeletal diseases.

Methods: Adults 65 years and older who presented to a tertiary rheumatologic hospital were included after informed consent. All patients recruited were assessed using the MAngable GeriatrIC Assessment (MAGIC) which addresses 14 common geriatric health problems. In addition, polypharmacy ( $\geq 5$ medication), muscle function using the Short Physical Performance Battery and frailty applying the Fried definition were assessed. Disability was quantified with the "Funktionsfragebogen Hannover" (FFbH), a validated tool for patients with rheumatologic diseases that can be easily converted to Health Assessment Questionnaire (HAQ) scores. Primary outcome was the frequency of the selected 17 geriatric health problems; the correlation of the total number of problems with $\mathrm{HAQ}$ scores was a secondary outcome.

Results: Of the 300 individuals included $67 \%$ were female with a mean age of $73 \pm 6.6$ years; $85 \%$ (> 50\% with rheumatoid arthritis) had a rheumatologic diagnosis. The remaining participants had either a chronic pain syndrome or degenerative joint/spine disease. On average participants had 7 out of 17 assessed geriatric problems. Females had more such problems than males (8 vs. $6, p<0.0001)$. Chronic pain and polypharmacy were most common but several others were also seen in more than $50 \%$ of patients (see Table). The mean HAQ Score was $1.67 \pm 0.79$. There was a positive correlation (see Graph) between the number of problems and the HAQ Score $\left(R^{2}=0.44, p<0.0001\right)$.

Conclusion: A systematic geriatric assessment can be successfully used to discover and quantify geriatric health problems in older patients with rheumatic and musculoskeletal diseases. These problems appear to be very common and importantly, patients with more problems had poorer functional status. Frailty, depression, incomplete vaccination status, cognitive impairment or polypharmacy are all known to negatively impact patient care. Recognizing and addressing geriatric problems has the potential to lead to health care improvements including adherence and medication side effects and might increase patient satisfaction and functional status independent of disease activity.

References:

[1] Buehring, B. and S. Barczi, Assessing the Aging Patient, in Spine Surgery in an Aging Population, N. Brooks and A. Strayer, Editors. 2019, Thieme: New York. p. 208.

[2] Cleutjens F, Boonen A, van Onna MGB. Geriatric syndromes in patients with rheumatoid arthritis: a literature overview. Clin Exp Rheumatol 2019;37(3):496-501

Geriatric Problem $\%$ present

Problems with Daily Activities

Problems with Vision

Problems with Hearing

Problems with Falls

Problems with Urinary Incontinence

Problems with Depression 
Geriatric Problem

$\%$ present

Lack of Social Support

Incomplete Vaccination

Problems with Cognition

Problems with Chronic Pain

Problems with Dizziness

Problems with Mobility

Problems with Unintentional Weight Loss

Inappropriate Medications present

Polypharmacy present

Frailty present

Short Physical Performance Battery low

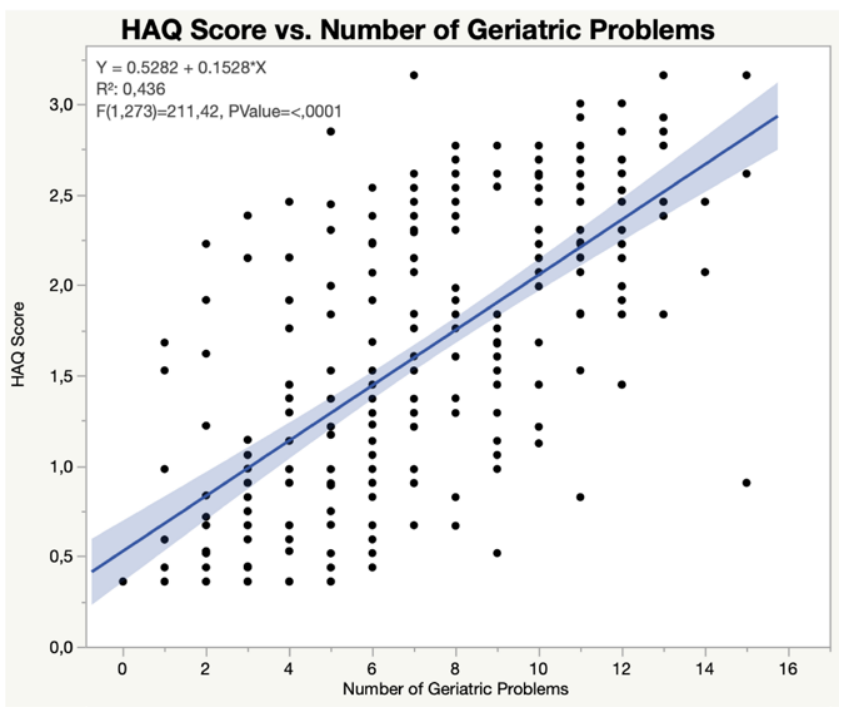

Acknowledgments: None

Disclosure of Interests: Anna Berrisch: None declared, loana Andreica: None declared, Styliani Tsiami: None declared, David Kiefer Grant/research support from: Novartis, Uta Kiltz Grant/research support from: AbbVie, Amgen, Biogen, Novartis, Pfizer, Consultant of: AbbVie, Biocad, Eli Lilly and Company, Grünenthal, Janssen, Novartis, Pfizer, UCB, Speakers bureau: AbbVie, MSD, Novartis, Pfizer, Roche, UCB, Xenofon Baraliakos Grant/research support from: Grant/ research support from: AbbVie, BMS, Celgene, Chugai, Merck, Novartis, Pfizer, UCB and Werfen, Consultant of: AbbVie, BMS, Celgene, Chugai, Merck, Novartis, Pfizer, UCB and Werfen, Speakers bureau: AbbVie, BMS, Celgene, Chugai, Merck, Novartis, Pfizer, UCB and Werfen, Juergen Braun Grant/research support from: Abbvie (Abbott), Amgen, BMS, Boehringer, Celgene, Celltrion, Centocor, Chugai, Eli Lilly and Company, Medac, MSD (Schering Plough), Mundipharma, Novartis, Pfizer (Wyeth), Roche, Sanofi- Aventis, and UCB Pharma, Consultant of: Abbvie (Abbott), Amgen, BMS, Boehringer, Celgene, Celltrion, Centocor, Chugai, EBEWE Pharma, Eli Lilly and Company, Medac, MSD (Schering-Plough), Mundipharma, Novartis, Pfizer (Wyeth), Roche, Sanofi-Aventis, and UCB Pharma, Speakers bureau: Abbvie (Abbott), Amgen, BMS, Boehringer, Celgene, Celltrion, Centocor, Chugai, EBEWE Pharma, Eli Lilly and Company, Medac, MSD (Schering-Plough), Mundipharma, Novartis, Pfizer (Wyeth), Roche, Sanofi-Aventis, and UCB Pharma, Bjoern Buehring Grant/research support from: GE/Lunar, Kinemed, Consultant of: Gilead, Abbvie, Lilly, GE/Lunar, Janssen, Amgen, Speakers bureau: UCB

DOI: 10.1136/annrheumdis-2020-eular.2815

\section{SAT0580 ANALYSIS OF ANAS/DFS70 PATTERN IN A LARGE COHORT OF AUTOIMMUNE/AUTOINFLAMMATORY DISEASES COMPARED WITH FIRST DEGREE RELATIVES AND HEALTHY CONTROLS EVALUATED IN A SINGLE HOSPITAL FROM COLOMBIA.}

C. Romero-Sánchez ${ }^{1,2,3}$, O. J. Calixto ${ }^{4}$, V. Romero- ${ }^{1,2}$, A. Vargas ${ }^{5}$, L. Castro ${ }^{6}$, J. Amador ${ }^{6}$, P. Lopez-Mojica ${ }^{6}$, D. Marin ${ }^{6}$, D. Acero-M ${ }^{2,7}$, M. Acevedo ${ }^{2,7}$, D. Rincón-Riaño ${ }^{1}$, J. M. Bello-Gualtero ${ }^{1,2} \cdot{ }^{1}$ Rheumatology and Immunology Department Hospital Militar Central, Bogota, Colombia; ${ }^{2}$ Clinical Immunology Group- Hospital Militar, School of Medicine, Universidad Militar Nueva Granada, Bogota, Colombia; ${ }^{3}$ Universidad El Bosque, Cellular and Immunology Group IInmuBo, Bogota, Colombia; ${ }^{4}$ Internal Medicine Department Hospital Militar Central, Universidad Militar Nueva Granada, Bogota, Colombia; ${ }^{5}$ Bacteriology degree, Faculty of Basic Sciences, Universidad Colegio Mayor de Cundinamarca, Bogota, Colombia; ${ }^{6}$ Dermatology Department Hospital
Militar Central, Universidad Militar Nueva Granada, Bogota, Colombia; ${ }^{7}$ Immunology Laboratory, Hospital Militar Central, Bogota, Colombia

Background: Autoimmune diseases have a broad phenotypic spectrum, with great variability in clinical manifestations. Anti-DFS70/LEDGFp75 (ANAS/ DFS70) antibodies have attracted interest as a positive result in patients without clinical evidence of autoimmune systemic rheumatic disease (SARD). It has been proven in non-rheumatic inflammatory diseases and in "apparently healthy" individuals.

Objectives: To assess ANAS/DFS70 performance in a large population with autoimmune/autoinflammatory diseases compared with first degree relatives and healthy controls.

Methods: A cross-sectional study was conducted. We analysed 531 individuals between 18-65 years old, 101 rheumatoid arthritis (RA) patients (ACR/EULAR 2010 classification criteria), 137 relatives from RA, 60 psoriasis (Ps) patients (Colombian classification consensus), 47 Undifferentiated connective tissue diseases(UCTD) patients and 186 healthy controls matched by age and sex. The healthy control group were individuals who lived and work similarly like those patients those criteria of exclusion criteria were to present autoimmune or auto-inflammatory disease, infectious, neoplasms, diabetes, antibiotic treatment, pregnancy or lactation, consanguinity with autoimmune entities. Ethical Committee approved.

The determination of ANAS-HEp2 antibodies (ANA-Hep-2 AESKU.Dignostic $®$, Autoantiboy test SYSTEM IMCO DIAGNOSTICS REF $1103 \otimes$ and ANA-Hep-2 AESKU.Dignostic $囚$ ) was carried out. The positive results (standard AC-2) are used as a confirmatory test the determination of ANAS / DFS70: AUTOANTIBOY TEST SYSTEM IMMCO DIAGNOSTICS (Knocked out, for the psip gene) REF $1108 \AA$ and CytoBead ANA Generic Assays ref $8065 \AA$ by indirect immunofluorescence-IFI technique. In addition, serum levels of C-reactive protein (PCR), erythrocyte sedimentation rate (ESR), IgG/lgA antibodies against citrullinated peptide (ACPA), and rheumatoid factor (RF). Absolute and relative frequencies were established.

Results: 531 participants were included: RA 19\%, 25,8\% RA relatives, Ps 11,3\%, UCTD $8,9 \%$, and $35 \%$ healthy controls. RA mean age was $41,8 \pm 12,2$ years,

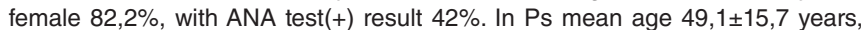
female $53,3 \%$, ANA test(+) $41,7 \%$. UCTD mean age $41,3 \pm 15,2$ years, female $85,1 \%$, and ANA test(+) $78,7 \%$. Relatives of RA mean age $38,7 \pm 12,2$ years female $73 \%$, ANA test(+) $26,3 \%$. And healthy controls mean age $41,3 \pm 12,2$ years, female $74,7 \%$, and ANA test(+) $26,9 \%$.

ANA/DFS70 was positive in a $6,4 \%$ in UCTD, $3,2 \%$ in healthy controls, $1,7 \%$ in Ps, $1,5 \%$ in Relatives of RA, no RA had positive results. These 12 participants were negative for acute phase reactants (ESR[-] 83,3\% and CRP[-] 66,6\%), as well as they were all negative for RF and two were positive for APCA from UCTD.

Conclusion: ANAS/DFS70 autoantibodies were present in very low frequency in patients with SARD. Thus, patients with a positive result tend to have a mild or non-progressing phenotype of autoimmune/inflammatory diseases, as UTCD. This is the first time ANA/DFS70 are tested in a large population cohort in Latin American countries which coincide with previous results in RA and RA relatives.

Acknowledgments: Hospital Militar Central-Universidad El Bosque-Immco diagnosis-Dizar Ltda and Generic Assay-Medipan.

Disclosure of Interests: Consuelo Romero-Sánchez: None declared, OmarJavier Calixto Employee of: Worked in Janssen Cilag as medical manager from 2016 to 2018, V Romero-A: None declared, A Vargas: None declared, Luis Castro: None declared, Julio Amador: None declared, Pedro Lopez-Mojica: None declared, Daniela Marin: None declared, Diana Acero-M: None declared, M Acevedo: None declared, Diana Rincón-Riaño: None declared, Juan Manuel Bello-Gualtero: None declared

DOI: 10.1136/annrheumdis-2020-eular.5082

\section{SAT0581 SERIOUS INFECTION RATES WITH BIOLOGICAL DISEASE MODIFYING ANTI-RHEUMATIC AGENTS (BDMARDS) AND PREDISPOSING FACTORS: A 5-YEAR RETROSPECTIVE REVIEW}

K. Celkys ${ }^{1}$, J. Ly ${ }^{1}$, M. Soden ${ }^{1} .{ }^{1}$ Townsville Hospital and Health Service, Townsville, Australia

Background: Biological and targeted synthetic disease modifying anti-rheumatic agents (bDMARDs) increase the risk of serious infections (SIs), however there is limited 'real-world' evidence comparing the relative risk of SI for individual bDMARDs. $(1,2)$

Objectives: This study examines the rates of Sls in a non-select Australian Northern Queensland (NQ) cohort of patients with various rheumatic diseases receiving treatment with a $b D M A R D$, to define predisposing factors and directly compare the bDMARDs.

Methods: A retrospective review was performed for all patients who received a bDMARD through the Townsville Hospital Rheumatology Department over the 